Journal of Immunological Methods, 33 (1980) 239-247

(c) Elsevier/North-Holland Biomedical Press

\title{
A 48-WELL MICRO CHEMOTAXIS ASSEMBLY FOR RAPID AND ACCURATE MEASUREMENT OF LEUKOCYTE MIGRATION
}

\author{
WERNER FALK, RICHARD H. GOODWIN, Jr. and EDWARD J. LEONARD \\ Immunopathology Section, Laboratory of Immunobiology, National Cancer Institute, \\ National Institutes of Health, Bethesda, MD 20205, and Neuroprobe Inc., 7621 Cabin \\ Road, Bethesda, MD 20034, U.S.A.
}

(Received 25 September 1979, accepted 21 November 1979)

\begin{abstract}
We designed a 48-well chemotaxis chamber to minimize manipulation time and amount of material required by the larger blindwell or Boyden chemotaxis chamber. Cell and chemoattractant dose-response curves showed that results were comparable to or better than those obtained with blindwell chambers. The volume of chemoattractant per well is $25 \mu \mathrm{l}$; the number of cells can be as low as 10,000. The time needed for setting up this multiwell unit and for staining the membrane filter sheet is negligible. Combined with the use of an image analyzer to count the number of migrated cells, the method is suitable for clinical research on the functional state of monocytes in large groups of patients.
\end{abstract}

\section{INTRODUCTION}

The first observation of chemotaxis was reported by Leber in 1888 . Since that time, there has been increasing interest in chemotaxis of polymorphonuclear leukocytes and macrophages, because a response to elaboration of chemotaxins may account for leukocyte accumulation at sites of inflammation. Chemotaxis has been studied in vitro on slides or coverslips (Commandon, 1917; McCutcheon et al., 1934; Harris, 1953), in capillary tubes (Ketchel and Favour, 1955), in culture wells under agarose (Cutler, 1974; Nelson et al., 1975; John and Sieber, 1976) and by a membrane filter technique first described by Boyden in 1962. Although the slide method is useful for specialized studies such as the tracking of individual leukocytes (Grimes and Barnes, 1973; Zigmond and Hirsch, 1973), Boyden's technique has the advantage of simplicity and the potential for providing quantitative data. It has been used in several clinical studies that have shown chemotaxis abnormalities in disease. The Boyden chamber consists of an upper and lower well separated by a membrane filter. Chemotactic solutions are placed in the lower well, cells are added to the top well, and after a period of incubation the cells that have migrated toward the chemotaxin are counted on the lower surface of the membrane. Both chamber and membrane have been modified by many investigators (Ward et al., 1965; Cornely, 1966; 
Keller and Sorkin, 1968; Tempel et al., 1970; Wilkinson, 1974; Jungi, 1975; Campbell, 1977; Valerius, 1978). A blindwell version (Boumsell and Meltzer, 1975) instead of the original Boyden chamber is now extensively used. However, despite modifications, the method is still too tedious and time consuming to allow handling of many samples. In addition to the space occupied in the incubator by the chambers, sample number is limited by the time required for assembling, disassembling, staining of individual membrane filters and washing of chambers. For clinical studies of chemotaxis and for increased accuracy, a simple method is needed which allows the processing of hundreds of samples. One step in this direction was made by Swanson (1977) who described a multichamber apparatus, consisting of 30 chambers in one unit. But it could not be used without centrifugation of the chamber and did not avoid the handling of single small filters. In this report, we describe results obtained with a newly designed 48-well chamber unit, consisting of top and bottom plates and a sealing gasket. Instead of multiple round filters, we use a single filter sheet. To conserve cells and chemotactic factor, the size of the wells has been reduced to a volume of $25 \mu \mathrm{l}$. The results are comparable to or better than those achieved with individual chambers, and assay time has been greatly reduced, due to manipulation of a single unit instead of 48 separate ones.

\section{MATERIALS AND METHODS}

\section{Cell preparation}

Venous blood was drawn into heparinized syringes from healthy donors and mononuclear cells were isolated by the method of $\mathrm{B} \phi \mathrm{yum}$ (1962). Total and differential counts were made for the final washed preparations. Differential counts were made with a fluorescence microscope after staining cell suspensions with euchrysin 3Rx (Roboz Surgical Instruments Co., Washington, DC). Cell suspensions contained 15-35\% monocytes, $65-85 \%$ lymphocytes and less than $1 \%$ granulocytes.

\section{Chemotactic factors}

N-formylmethionyl-leucyl-phenylalanine-methylester (referred to as peptide) was a generous gift of Dr. P.K. Ho (Lilly Co., Indianapolis). Human serum derived complement component $\mathrm{C} 5 \mathrm{a}$ and lymphocyte derived chemotactic factor (LDCF) were prepared as described (Falk and Leonard, 1980). The pooled activity peak of a Sephadex G-100 fractionation of the crude factor was concentrated $10 \times$ by means of an immersible molecular separation unit (Millipore Corp., Bedford, MA).

\section{Description of the chambers}

We constructed two kinds of multiwell chamber, one 24-well chamber with $200 \mu \mathrm{l}$ per well volume and a 48-well chamber with $25 \mu$ l volume (Fig. 1). The single units of the 24 -well chamber were exactly equivalent to 


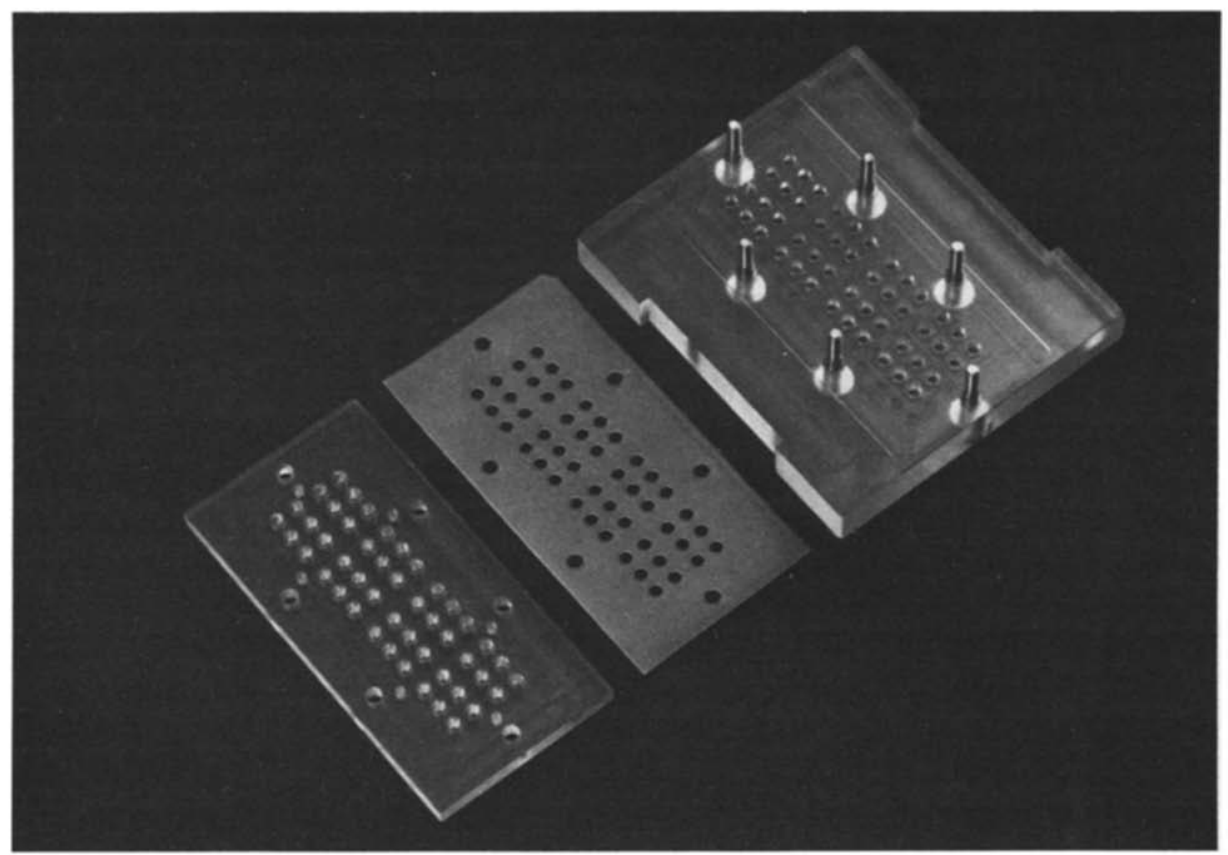

Fig. 1. Component parts of the 48-well micro chemotaxis chamber. Upper right: bottom plate with 48 wells of $25 \mu$ l volume each. The wells have raised rims to prevent spilling of chemotactic fluids from one well to another. The bolts are tapered to allow for exact positioning and easy assembling of the other components. A $7.6 \mathrm{~cm} \times 2.5 \mathrm{~cm}$ Nucleopore filter sheet is placed on the filled wells. Middle: the silicon gasket, which is slipped over the bolts and placed on the filter. Lower left: the top plate, bottom side up. The 48 holes (maximal volume $50 \mu \mathrm{l}$ ) have raised rims like the bottom wells. The top plate is pressed down by knurled nuts. The assembled apparatus is shown in Fig. 2.

blindwell chambers and this set up was only used for comparison with the blindwell chamber. It will not be discussed in detail. The 48 -well chamber (Figs. 1 and 2) consists of a bottom plate with forty-eight $25 \mu \mathrm{l}$ wells. A silicon gasket is used to obtain a perfect seal. The holes in the top plate become the top chambers after assembling. Nucleopore filter sheets of $5 \mu \mathrm{m}$ hole size and about $10 \mu \mathrm{m}$ in thickness were placed between bottom plate and gasket. The sheet size was $7.6 \times 2.5 \mathrm{~cm}$. The top and bottom wells have slightly raised rims to eliminate any chance of movement of chemotactic solutions from one well into another. The distance between the wells is $2 \mathrm{~mm}$. The filter area used per single chamber is $8 \mathrm{~mm}^{2}$ and the ratio of this area to the lower well volume is $1: 3$ in contrast to $1: 4$ for the normal 200 $\mu \mathrm{l}$ blindwell chamber. Six threaded posts with knurled nuts are spaced along the edges of the apparatus to supply an evenly distributed pressure. No leakage was observed. The layout of the chamber allows for duplicates, triplicates or quadruplicates. The experiments described in this paper were per- 


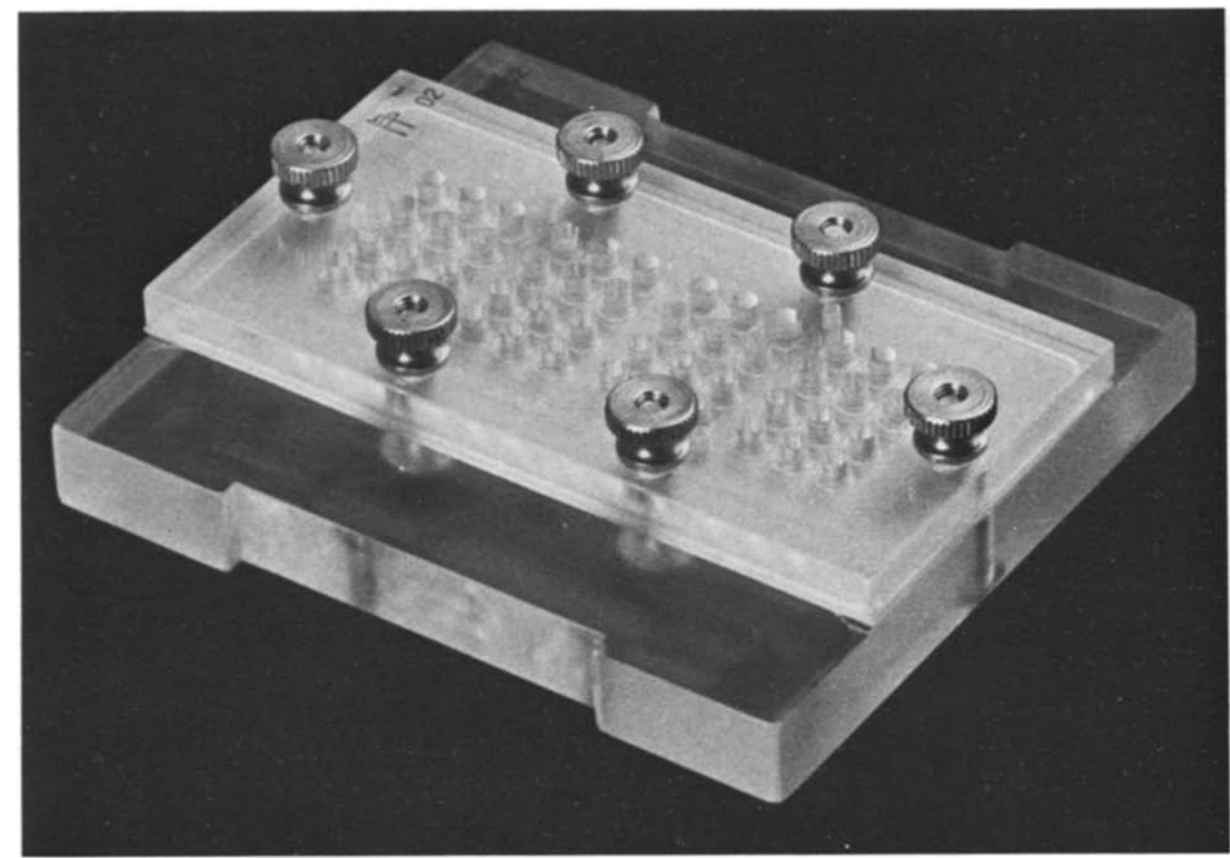

Fig. 2. The assembled 48-well micro chemotaxis chamber. This is a photograph of the original prototype. Production models will be the same except for minor modifications.

formed with a prototype chamber. Production chambers will be available from Neuro Probe.

\section{Chemotaxis assay}

The assay with blindwell chambers was carried out as previously described (Falk and Leonard, 1980). Briefly, $200 \mu \mathrm{l}$ of chemotactic solution was placed in the bottom wells and the top wells were filled with $0.3 \mathrm{ml}$ of Gey's balanced salt solution containing $2 \%$ BSA (Gey's BSA) and $8 \times 10^{4}$ monocytes. The two chambers were separated by a polycarbonate filter with $5 \mu \mathrm{m}$ holes (Nucleopore, Pleasanton, CA). The chambers were incubated for $2 \mathrm{~h}$ at $37^{\circ} \mathrm{C}$ in humidified air with $5 \% \mathrm{CO}_{2}$; the filters were removed, stained and the migrated cells were counted.

The procedure for the multiwell chamber was similar. The bottom wells were filled with $25 \mu$ l of chemotactic solutions in Gey's BSA. Dilutions were prepared in microtiter plates. A filter sheet was then picked up by forceps and carefully placed on the bottom plate. Gasket and top plate were slipped over the threaded posts shown in Fig. 1 and bolted down. The monocyte suspension $(30 \mu \mathrm{l})$ was added to the top chambers and the apparatus was incubated for $2 \mathrm{~h}$. For removal of non-migrated cells from the top side of the filter at the end of the incubation, the chamber was disassembled and the 
filter was suspended between 2 clamps. The filter was then drawn gently up the edge of a windshield wiper blade. This was done twice, with immersion in phosphate-buffered saline between wipings. The filter was then fixed for about $2 \mathrm{~min}$ in methanol, mounted on a glass slide and dried. The cells on the mounted sheet were stained with Diff-Quick (Harleco, Gibbstown, NJ). Counting of the migrated cells was done in different ways. Filters of blindwell chambers were counted as previously described (Boetcher and Leonard, 1974). Migrated cells on filter sheets were evaluated by means of an Optomax Image analyzer (Optomax Inc., Hollis, NH). The area covered by migrated cells per selected field was measured. Five fields were sampled per replicate, with a $25 \times$ objective. In another experiment, with lower cell numbers, a single $25 \times$ field per replicate was counted by eye. All assays were done in triplicate. All manipulations, including staining and washing the chamber took about $10 \mathrm{~min}$.

\section{RESULTS}

\section{Comparison with blindwell chambers}

Using the blindwell chamber assay as a standard, we compared the dose responses of human monocytes to human $\mathrm{C5a}$ and chemotactic peptide in blindwell and 24-multiwell chambers. As shown in Fig. 3, the responses were not significantly different. The slight differences seen in the figures were not consistent.

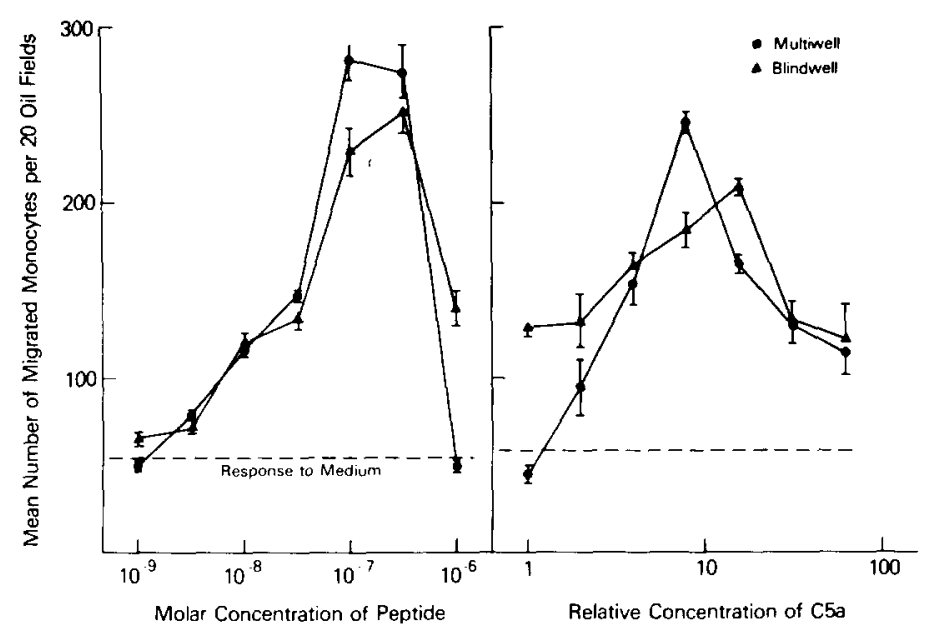

Fig. 3. Comparison of dose responses of human monocytes to chemotactic peptide and human C5A in a blindwell and a multiwell chamber. A 24-well multichamber with the same dimensions as the blindwell chamber was used. The left panel shows the response to $\mathrm{N}$-formyl-methionyl-leucyl-phenylalanine methylester. The right panel shows the response to human serum derived purified C5a. The same filter lot was used in both chambers. The error bars represent the standard error of the mean of triplicates. A relative concentration of 1 means a dilution of $1: 6400$ of the C5a stock solution. 


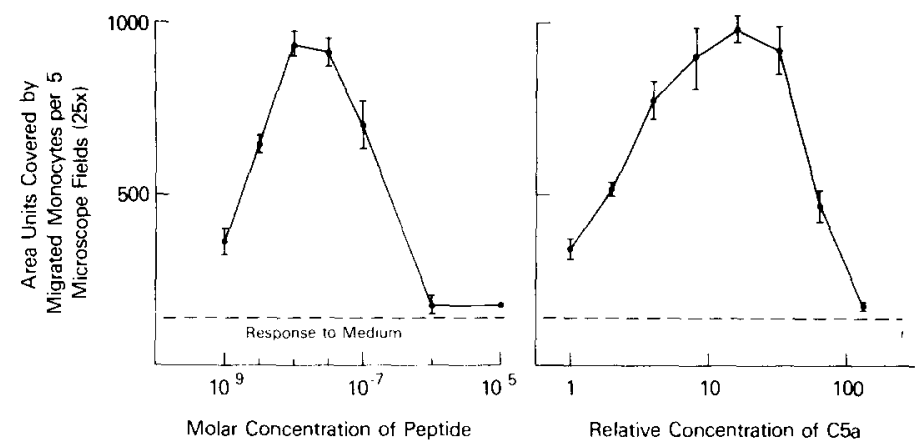

Fig. 4. Dose responses in a micro-multiwell chamber of human monocytes to chemotactic peptide and human C5a. The peptide was N-formyl-methionyl-leucyl-phenylalaninemethylester; $\mathrm{C} 5 \mathrm{a}$ was prepared from human serum. The area covered by migrated cells was measured with an image analyzer; the area units are arbitrary. The error bars represent the standard error of the mean of triplicates. A relative concentration of 1 means a dilution of $1: 6400$ of the $\mathrm{C} 5$ a stock solution. The input monocyte number per well was 70,000 .

\section{The 48-well microchamber}

After showing that results with the 24-well and individual blindwell chambers were comparable, we designed a 48-well unit with decreased well volumes in order to further reduce manipulation time and to conserve cells and chemotactic factors. The effective filter area of a blindwell chamber is about $50 \mathrm{~mm}^{2}$, in contrast to 8 for the microchamber. We therefore were

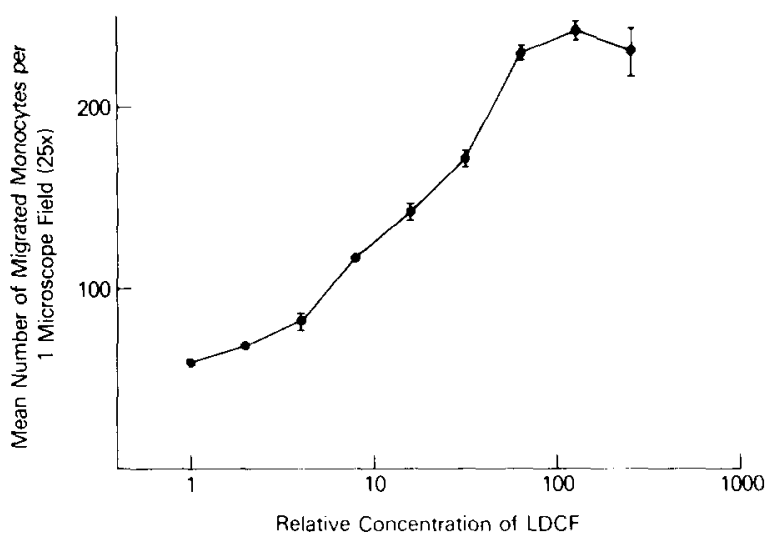

Fig. 5. Dose response of human monocytes to human lymphocyte derived chemotactic factor in a micro-multiwell chamber. LDCF was prepared as described in Materials and Methods. The error bars represent the standard error of the mean of triplicates. One field per replicate was counted by eye. No error bar means that the standard error was smaller than the dimension of the point. Relative concentration of 1 means a dilution of $1: 512$ of the stock solution. The input monocyte number per well was 36,000 . 


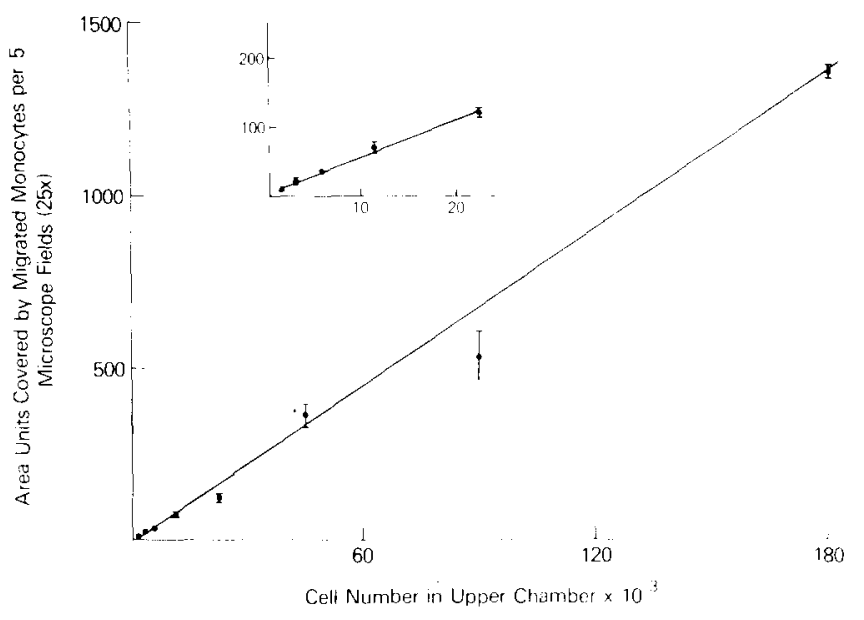

Fig. 6. Cell-dose response of human monocytes in a micro-multiwell chamber. The number of monocytes added to the upper chamber is shown on the abscissa. N-formylmethionyl-leucyl-phenylalaninemethylester $\left(10^{-7} \mathrm{M}\right)$ was used as attractant. The insert represents the lower part of the curve on a expanded scale. The area units are arbitrary and were determined with an image analyzer. The error bars represent the standard error of the mean of triplicates. No error bar means that the standard error was smaller than the dimension of the point.

able to reduce the bottom well volume to $25 \mu \mathrm{l}$ and the top well volume to $30 \mu \mathrm{l}$. We also needed only $1 / 8$ the number of cells to obtain the same cell density on the filter as for the blindwell chamber. The dose responses to peptide (Fig. 4, left panel), C5a (Fig. 4, right panel) and LDCF (Fig. 5) showed that the assay is sensitive to changes in concentration of chemotactic agents. The cell dose response (Fig. 6) showed the same wide range of linearity as we showed earlier for the blindwell chamber. The distribution of the cells was surprisingly homogeneous, in contrast to filters of blindwell chambers. In addition, variation among triplicates seemed to be less in the multiwell unit than with individual chambers. This may be due to the small size providing an equal microenvironment for all single chamber units during incubation.

\section{DISCUSSION}

Chemotaxis in vitro of leukocytes of man and experimental animals is a widely used functional assay. One of its limitations has been the experimental procedure, which allowed the processing of only a limited number of samples. In this paper we describe a greatly improved procedure to measure chemotaxis. The various dose responses show the reliability of the assay. The system works as well for mouse macrophages (data not shown).

The micro-multiwell chamber offers a number of important advantages 
over other chemotaxis methods. The time to perform a large assay is negligible. The volume of chemotactic factor needed is very low $(0.075 \mathrm{ml}$ per triplicate), and the cell number needed per chamber can be as low as 10,000. We think in addition that the small size and the therefore more uniform conditions in the incubator make the use of triplicates unnecessary. This would further decrease working and counting time. We know of only one published multichamber assay (Swanson, 1977); but it had disadvantages, such as the unexplained need for centrifugation and the use of single filters. If necessary, our chambers can be centrifuged in a Beckman TJ-6 centrifuge. The chamber is not restricted to the use of Nucleopore filters; with modifications cellulose filters or double filter techniques could also be used.

To achieve this excellent performance, attention to critical design details and high precision in construction were necessary. Special cutting tools of high quality were made. The lucite blocks were milled to provide small raised rims around each well to prevent movement of chemotactic factor solution from one well to another. The 6 bolts were made not only to provide the tie downs, but also a perfect alignment of top and bottom wells. This was achieved by tapering. It is important to treat the multiwell chamber as a precision instrument in the general handling, rinsing and air drying of the component parts. We recommend air drying at room temperature. Oven drying or use of organic solvents may damage the materials.

This assay system makes it possible to use chemotaxis as a clinical test, because $2-5 \mathrm{ml}$ of blood are sufficient to do a large assay that includes doseresponse curves to several different chemotaxins in a short time. The other limitation on the accumulation of chemotaxis data is the counting of migrated cells. Since this is tiresome and time consuming, some investigators have used radioactive labeling methods (Gallin et al., 1973; Papierniak et al., 1976). However, fast and reliable counting of stained cells on or in chemotaxis filters, without the additional step of radioactive labeling, is now possible by the use of image analyzers. With the Optomax analyzer, we counted cells by number and by area covered by the cells. Both methods gave comparable results and are very fast.

In summary, the combined use of our new micro-multiwell chamber and of the image analyzer makes chemotaxis a rapid and simple assay. We think that it can now be used in the clinic to obtain information about the functional state of leukocytes.

\section{REFERENCES}

Boetcher, D.A. and E.J. Leonard, 1974, J. Natl. Cancer Inst. 52, 1091.

Boumsell, L. and M.S. Meltzer, 1975, J. Immunol. Methods 115, 1746.

B фyum, A., 1962, J. Exp. Med. 115, 453.

Campbell, P.B., 1977, J. Lab. Clin. Med. 90, 381.

Commandon, J., 1917, C.R. Soc. Biol. 80, 314.

Cornely, H.P., 1966, Proc. Soc. Exp. Biol. Med. 122, 831.

Cutler, J.E., 1974, Proc. Soc. Exp. Biol. Med. 147, 471. 
Falk, W. and E.J. Leonard, 1980, Submitted for publication.

Gallin, J.E., R.A. Clark and H.R. Kimball, 1973, J. Immunol. 110, 233.

Grimes, G.J. and F.S. Barnes, 1973, Exp. Cell Res. 79, 375.

Harris, J., 1953, J. Pathol. Bacteriol. 66, 135.

John, T.J. and O.F. Sieber, Jr., 1976, Life Sci. 18, 177.

Jungi, T.W., 1975, Int. Arch. Allergy Appl. Immunol. 48, 341.

Keller, H.U. and E. Sorkin, 1968, Experientia 24, 641.

Ketchel, M.K. and C.B. Favour, 1955, J. Exp. Med. 101, 647.

Leber, T., 1888, Fortschr. Med. 6, 460.

McCutcheon, M., W.B. Wartman and H.M. Dixon, 1934, Arch. Pathol. 17, 607.

Nelson, R.D., P.G. Quie and R.L. Simmons, 1975, J. Immunol. 115, 1650.

Papierniak, C.K., R.E. Bourey, R.R. Kretschmer, S.P. Gotoff and L.G. Colombetti, 1976, J. Nucl. Med. 17, 988.

Swanson, M.J., 1977, J. Immunol. Methods 16, 385.

Tempel, T.R., R. Snyderman, H.V. Jordan and S.E. Mergenhagen, 1970, J. Periodont. $41,71$.

Valerius, N.H., 1978, Acta Pathol. Microbiol. Scand. 86B, 149.

Ward, P.A., C.G. Cochrane and H.J. Müller-Eberhard, 1965, J. Exp. Med. 122, 327.

Wilkinson, P.C., 1974, Chemotaxis and Inflammation (Churchill-Livingstone, London) p. 42.

Zigmond, S.H. and J.G. Hirsch, 1973, J. Exp. Med. 137, 187. 